\title{
Not only bupivacaine but also propofol is sinking in lipid?
}

\author{
Yushi U. Adachi • Atsushi Numaguchi • \\ Naoyuki Matsuda
}

Received: 27 September 2013/ Accepted: 28 September 2013/Published online: 16 October 2013

(C) Japanese Society of Anesthesiologists 2013

Keywords Propofol $\cdot$ Lipid emulsion $\cdot$ Central nervous system

To the Editor:

We read with great interest the recent article [1] and editorial [2] by Oda and Ikeda in this journal. The authors clearly demonstrated the effect of lipid emulsion on central nervous system abnormalities induced by bupivacaine toxicity using the unique experimental in vivo animal model. Although many anesthesiologists in developed countries, including Japan, would prefer to choose safer and long-acting local anesthetics, e.g., ropivacaine, basic research on the mechanism of drug toxicity and exploration of treatment for intoxication are absolutely important.

Oda concluded that a lipid sink resulting from administration of the emulsion appears to play an important role for various lipophilic agents. Recently, we demonstrated that the hypnotic activity of propofol, a popular intravenous general anesthetic and one of the most lipophilic drugs, was diminished by simultaneous injection of lipid as a solvent [3]. Injection site pain of propofol is widely known and is considered to result from the increase in free aqueous fraction of the drug [4]. The coadministration of lipid solution could alleviate this pain by the decrease in free propofol caused by the lipid sink. Therefore, we hypothesized that not only injection pain but also the effect of the drugs on the central nervous system might be modified by lipid injection, similar to bupivacaine neurotoxicity.

Lipid rescue is reported as effective for many other drugs besides bupivacaine. The precise mechanism is not fully understood, and further studies are required.

Conflict of interest All authors have no conflict of interest concerning the current letter.

\section{References}

1. Oda Y, Ikeda Y. Effect of lipid emulsion on the central nervous system and cardiac toxicity of bupivacaine and levobupivacaine in awake rats. J Anesth. 2013;27:500-4.

2. Oda Y. Lipid resuscitation: development in basic research and application to clinical practice. J Anesth 2013 [in press].

3. Adachi Y, Tochikubo J, Tamura T, Hashimoto S, Hatano T, Matsuda N. The hypnotic activity of propofol formulation was enhanced by dilution with crystalloid solution at the induction of anaesthesia in ddY mice. Eur J Anaesthesiol. 2013;30(e-suppl 51):149-50 (9AP4-P5).

4. Doenicke AW, Roizen MF, Rau J, Kellermann W, Babl J. Reducing pain during propofol injection: the role of the solvent. Anesth Analg. 1996;82:472-4.

Y. U. Adachi $(\bowtie) \cdot$ A. Numaguchi

Department of Emergency Medicine, Nagoya University

Hospital, 65 Tsurumai-cho, Showa-ku, Nagoya 466-8550, Japan

e-mail: yuadachi@med.nagoya-u.ac.jp

N. Matsuda

Department of Emergency and Critical Care Medicine, Nagoya

University Graduate School of Medicine, Nagoya, Japan 